\title{
A Mobile Frequency Allocation Algorithm Based on the Graph Theory
}

\author{
L. Yang, Y.H. Yu \\ Department of Telecommun ication Engineering \\ Jilin University \\ Jilin, China
}

\begin{abstract}
By using the theory of point coloring in graph theory, the algorithm in this article converts the relationship among base stations to the relationship between the vertices and lines in a graph, performs the point coloring for the great connecting diagram on the basis of the thought of the wheel diagram, and then determines whether to complete frequency allocation by certain constraint conditions. In additional, we consider a mapping relation among the sets of vertices and frequency points, so as to reduce the same frequency interference among the cells and improve the quality of communication. Based on the analysis on the example of Yanji, the results show that this optimizing mobile frequency allocation method can effectively improve the robustness of the communication, decrease the same frequency interference between the base stations, and achieve the improvement of communication quality.
\end{abstract}

Keywords-frequency configuration; graph theory; vertex coloring; same frequency interference

\section{INTRODUCTION}

The coloring of graphs plays a role in graph theory. The origin of the coloring of graphs is famous four color conjecture. In 1852, Guthirie brothers put forward four-color problem about the communication, later developing the famous 4-coloring algorithm now. Graph coloring problem has wide applications in the realm of other sciences, such as the problem about the timetable, labor division, transportation and so on. Many practical problems can be converted to graph coloring problem. Its main idea is that a network topology which consis ts of base stations is abstracted into a graphic model, and then establishing the wheel figure model according to the point coloring model in graph theory we realize configuration problems of the frequency points.

The frequency configuration for the mobile communication systems has become one of the important technologies of mobile communication research. In recent years, mobile communications business in China constantly expands the scales, and the forms of mobile communication are developing daily. Then, the 4G technology has been promoted and operated. In this case, accounting for the optimization of wireless network operating environment and the best optimal coverage of various typical frequencies in mobile communication networks, the guarantee for network convenience and safety of operation has become a main problem for communication development in the future. Take example for TD-SCDMA network, its construction and development always go along with actual network test. Through testing to master network performance and find ing the existing problems, and then in view of the problem we seek the best optimal method to solve the problem step by step and achieve the purpose about the network optimization. Moreover, there are a large number of researchers who committed to the research from several aspects to improve the quality of the communication in mobile cellular network, so many frequency point allocating schemes for base stations are proposed. For example, the authors of references $[1,2,3]$ studied the frequency allocating schemes based on the swarm intelligence algorithm respectively, which were combined with frequency point distribution model, reducing the same frequency interference between the base stations .Recently, the interference figure of adaptive subband allocation algorithm presented in reference [4] to deal with the problem of subband distribution for femtocells, reduces the interference between macrocells and femtocells. The cognitive spectrum allocation algorithm based on graph coloring is proposed reference [5], which minimizes the interference between the base stations. In reference [6], the author proposes a new method of dynamic spectrum allocation to reduce the interference in the systems and improve the utilization of the frequency points. The researches on the distribution of frequency points for cellular base stations have improved the communication quality. However, the algorithm robustness remains improved further because of obvious redundancy phenomenon for existing mobile frequency allocation algorithm. Due to some shortcomings (such as large same frequency interference, bad robustness) for the existing methods of mobile frequency distribution, this paper proposes a mobile frequency allocation method based on graph theory.

\section{SYSTEM MODEL}

Converting the adjoining relationship between the base stations into a connection graph in graph theory, the connection graph which is called base station connection graph is denoted by $\mathrm{G}(\mathrm{V}, \mathrm{E})$. The set $\mathrm{V}$ is a non-empty vertex set, the set $\mathrm{E}$ (called the edge set of graph G) is an edge set constituted by disorder pairs of different elements in the set $\mathrm{V}$, where the base stations are designed into a set of vertices $\mathrm{V}$ in the connection graph, and adjoining relations are designed into the edge set $\mathrm{E}$. Consequently, frequency optimization scheme can be transformed into vertex coloring for the connection graph. The corresponding relations between the connection graph of base stations and mobile communication parameters can be expressed as shown in table 1. 
TABLE I. CORRESPONDING RELATIONS BETWEEN THE CONNECTION GRAPH OF BASE STATIONS AND MOBILE COMMUNICATION PARAMETERS

\begin{tabular}{|l|l|}
\hline $\begin{array}{l}\text { Graph theory } \\
\text { paramet ers }\end{array}$ & Mobile communication optimization parameters \\
\hline Dyeing number & Frequency point number \\
\hline Dyeing & Frequency point \\
\hline Vertex set & Base station set \\
\hline Edge set & Adjoining cell set \\
\hline
\end{tabular}

The edges and vertices in the base station connection graph are divided into two sets, namely the greatest set and the limit set, respectively denoted by $E_{1}$ and $E_{2}$. The principle which is divided is that the greatest set $E_{1}$ contains the most edges and the vertices connected by these edges under the condition excepting the intersections among the vertices and all edges without other intersection points in one set. The limit set $E_{2}$ is the rest edges which are removed from the greatest set in the connection graph and vertices connected by the edges.

The graph of the greatest set $E_{1}$ is completed into the greatest connection graph. As a result, a wheel graph model which is equal to the number of boundary points is derived.

$$
\left.L_{m}^{k}\right|_{b_{k}}\left(\mathrm{a}_{1}, \mathrm{a}_{2}, \cdots, \mathrm{a}_{m}\right)=\left\{\begin{array}{l}
b_{k} a_{1} a_{2} a_{3}(m=3) \\
b_{k} \overline{a_{1}} \overline{a_{2}} \overline{a_{3}} \overline{a_{4}}+b_{k} \overline{a_{1}} a_{2} \overline{a_{3}} a_{4}+b_{k} a_{1} \overline{a_{2}} a_{3} \overline{a_{4}}(m=4) \\
b_{k} a_{m} L_{m-1}^{k}\left(\overline{a_{1}}, a_{2}, \cdots, a_{m-2}, \overline{a_{m-1}}\right)+b_{k} \overline{a_{\mathrm{m}}} a_{m-1} L_{m-2}^{k}\left(\overline{a_{1}}, a_{2}, \cdots, a_{m-2}\right)+b_{k} \overline{a_{\mathrm{m}}} \overline{a_{\mathrm{m}-1}} L_{m-2}^{k}\left(a_{1}, a_{2}, \cdots, a_{m-2}\right)(m \geq 5)
\end{array}\right.
$$

where the number of wheel graphs is denoted by $\mathrm{k}, \mathrm{m}$ denotes Boolean space dimension, $a$ is expressed as a Boolean variable, $\bar{a}$ flips the value of the variable $a$. And the $a_{i}=1$ expresses that the $\mathrm{i}$ th non center vertex with the center vertex are different when coloring, $\overline{\boldsymbol{a}}_{i}=\mathrm{O}$, and vice versa. where $i=1,2, \cdots, m$. The variable $b_{k}$ is keying coefficient. In general, the number of frequency points for configuration is more than four. Therefore, the connection graph can not use the simple 4-coloring. So in order to use frequency point more effectively, the authors introduce the keying coefficient, which divides all frequency points into several sets consisting of four elements.

Because all sets of the 4-coloring for the great connection graph and a subset of the $\mathrm{n}$ dimension Boolean vector space $\mathrm{V}$ are of one to one correspondence, the vertex coloring problem can be converted to Boolean equation problem. Then define a Boolean function on the $\mathrm{n}$ dimension Boolean vector space $\mathrm{V}$, which is written as:

$$
\left.L_{m}^{k}\right|_{b_{k}}\left(\mathrm{a}_{1}, \mathrm{a}_{2}, \ldots, \mathrm{a}_{m}\right)= \begin{cases}1, & \text { each child wheel graph model can get the correct point coloring } \\ 0, & \text { else }\end{cases}
$$

Then, on the basis of Boolean function we can determine whether each wheel graph succeeds in point coloring.As mentioned above, isolated graph consisting of $\mathrm{n}$ nodes can be represented by a wheel graph model, which can be mathematically written as $\mathbf{L}=\left\{\left.\mathbf{L}_{\mathrm{m}_{\mathrm{n}}}^{1}\right|_{\mathrm{b}_{\mathrm{k}}},\left.\mathbf{L}_{\mathrm{m}_{2}}^{2}\right|_{\mathrm{b}_{\mathrm{k}}}, \ldots \ldots,\left.\quad \mathbf{L}_{\mathrm{m}_{\mathrm{n}}}^{\mathrm{n}}\right|_{\mathrm{b}_{\mathrm{k}}}\right\}$.If the network can
And then, considering vertex coloring method in wheel graph theory we perform point coloring for vertexes in the greatest set.

\section{SIMULATION MODEL}

\section{A. Algorithm Basic Idea}

The completed greatest connection graph is made up of several wheel graph models and each non-boundary vertex is a center vertex in a wheel graph model, so we can use 4-coloring for the greatest plane graph $[7,8,9]$ theory and Boolean equations to complete the vertex coloring for each wheel graph models.

\section{B. Solution by the Calculation Using Boolean Equations and 4-Coloring Theories}

Though Boolean equations [10,11] and constraint conditions, the recursive formula for the vertex coloring is expressed as follows: complete point coloring, then satisfy the equation $L=1$, That is, each child wheel graph model can get the correct point coloring. The coloring problem that the greatest connection graph is completed can be judged by

$$
\left.\mathbf{L}_{m_{n}}^{1}\right|_{b_{k}}=\left.\mathbf{L}_{m_{2}}^{2}\right|_{b_{k}}=\cdots \cdots=\left.L_{m_{n}}^{n}\right|_{b_{k}}=1
$$

\section{Frequency Point Distribution Method for the Whole Network}

Considering many vertex coloring schemes which are obtained from the subsection 3.2 for the greatest connection graph, and then the authors act the limit set $E_{2}$ as constraint conditions, and judge that the vertex coloring scheme among the greatest set $E_{1}$ is reasonable or not. Thus, we can select vertex coloring schemes which are suitable for the whole base station connection graph.

The limit set also meet the mapping relationship of the connection graph. If the base station connection graph G completes the normal frequency point configuration, the frequency distribution for cells [12] meet mapping relationship between the frequency points and vertices in the connection graph. As a result, the vertex set $\mathrm{V}$ in the graph $\mathrm{G}$ corresponds and maps to the set $\mathrm{P}$ of frequency distribution for cells, namely $\mathbf{V}(\mathbf{G}) \rightarrow \mathbf{P}(\mathbf{G})$. If an edge in the base station connection graph $\mathrm{G}$ belongs to the limit set, and the vertex $\mathrm{u}$ and vertex $\mathrm{v}$ is respectively two vertices of the edge e, and considering the principle that the adjacent vertexes can't use 
the same color, namely $\mathbf{P}(\boldsymbol{u}) \neq \mathbf{P}(v)$, we can obtain the vertex coloring schemes for the connection graph. Therefore, the equations of vertex coloring for the whole base station connection graph can be calculated as (4),


meeting with the solution of the eqn. (4), it can be confirmed that the vertex coloring scheme for the connection graph are obtained.

$$
\left\{\begin{array}{l}
\left.L_{m_{n}}^{1}\right|_{b_{k}}=1 \\
\left.L_{m_{2}}^{2}\right|_{b_{k}}=1 \\
\cdot \\
\cdot \\
\left.L_{m_{n}}^{n}\right|_{b_{k}}=1 \\
P\left(v_{l}\right) \neq P\left(u_{l}\right)
\end{array}\right.
$$

\section{Algorithm Procedure}

The frequency scheme which is constrained by the limit set $E_{2}$ to coordinate each keying wheel graph makes the frequency optimization in the whole network. Considering for the mapping relationship between the keying coefficient $b_{k}$ and the set $\mathrm{P}$ is crucial before modifying the keying coefficient $b_{k}$ in a wheel graph. The flow chart of the algorithm is shown in fig. 1 .

\section{ANALYSIS OF APPLICATION}

In order to analyze the performance of the frequency allocation algorithm based on graph theory, in this case, this paper takes example of the application of mobile communication frequency configuration at walking streets in Yanji. Then the effectiveness of the proposed algorithm is verified. First of all, the data for the wireles s communication in grids is extracted. Consider that the way is the road test (DT), and the average speed is 25 kilometers per hour. After testing we can find that, the biggest traffic throughout is in the center of the west square in Yanji whose square is nearly 2300 square meters.

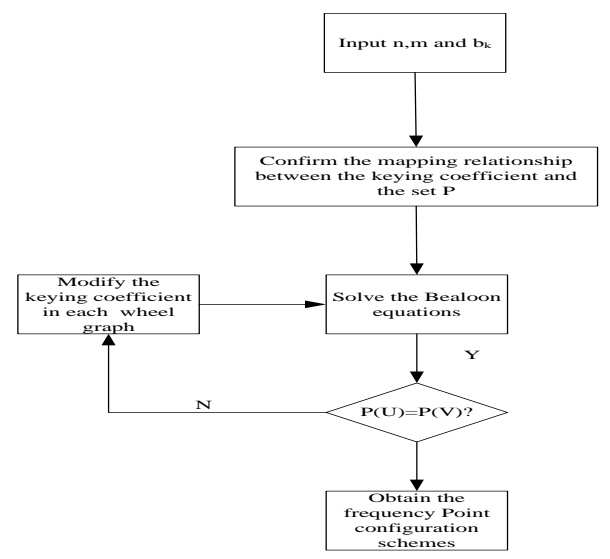

FIGURE I. THE FLOW CHART FORTHE MOBILE FREQUENCY ALLOCATIONALGORITHM BASED ON GRAPH THEORY



FIGURE II. BASE STATION CONNECTION GRAPH

As shown in fig. 3, through the analys is it can be found that, due to there are a large amount of customers during the early peak flow, the base station density is higher. In this case, the workers for the optimal network use batch frequency point software to optimize the frequency point, which can lead to the stronger same frequency interference under the TD-SCDMA channels (the areas denoted by red are less $\mathrm{C} / \mathrm{I}$, green for the areas where is normal C/I, blue for the areas where is normal $\mathrm{C} / \mathrm{I})$. Then, we use the mobile frequency configuration optimization algorithm based on graph theory to make TD-SCDMA frequency point configuration for street areas in figure and area engineering optimization.

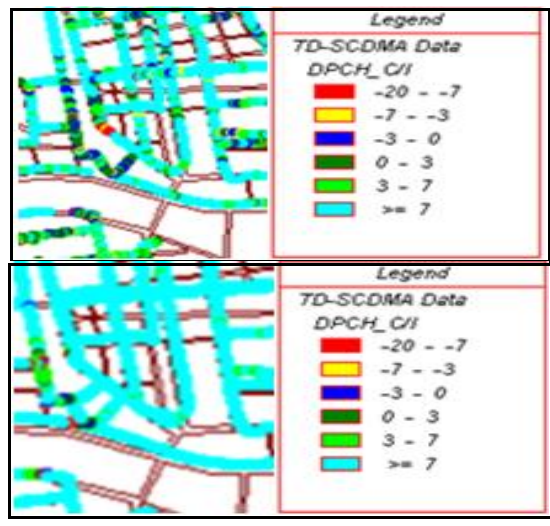

FIGURE III. NETWORK C/I BEFORE FREQUENCY POINT OPTIMIZED FIGURE 4: NETWORK C/I AFTER FREQUENCY POINT OPTIMIZED

TABLE II. NUMBER OF ADJACENT CELLS IN EACH CELL

\begin{tabular}{|c|c|c|c|c|c|c|c|c|c|c|c|c|}
\hline $\mathrm{n}$ & $\mathrm{m} 1$ & $\mathrm{~m} 2$ & $\mathrm{~m} 3$ & $\mathrm{~m} 4$ & $\mathrm{~m} 5$ & $\mathrm{~m} 6$ & $\mathrm{~m} 7$ & $\mathrm{~m} 8$ & $\mathrm{~m} 9$ & $\mathrm{~m} 10$ & $\mathrm{~m} 11$ & $\mathrm{~m} 12$ \\
\hline 12 & 5 & 6 & 4 & 5 & 5 & 7 & 5 & 5 & 5 & 4 & 7 & 5 \\
\hline
\end{tabular}

TABLE III. MAPPING RELATIONSHIP BETWEEN THE KEYING COEFFICIENT AND FREQUENCY POINT SET

\begin{tabular}{|c|c|c|c|c|}
\hline$b_{k}=1$ & $P 1=\{10080$ & 10088 , & 10096, & $10104\}$ \\
\hline$b_{k}=-1$ & $P 2=\{10096$ & 10104, & 10112, & $10120\}$ \\
\hline
\end{tabular}

TABLE IV. CALCULATION RESULT S AND FREQUENCY POINT OPTIMIZATION SCHEME

\begin{tabular}{|l|l|l|l|l|l|l|l|l|l|l|l|}
\hline \multicolumn{2}{|l|}{$b_{k}=1$} & 1 \\
\hline $\mathrm{v} 1$ & 12 & $\mathrm{v} 4$ & 5 & $\mathrm{v} 6$ & $\mathrm{v} 7$ & $\mathrm{v} 8$ & $\mathrm{v} 10$ & $\mathrm{v} 11$ & $\mathrm{v}$ & $\mathrm{v} 9$ & $\mathrm{v} 12$ \\
\hline 1008 & 0104 & 10088 & 10096 & 10088 & 10080 & 10096 & 10088 & 10104 & 10112 & 10112 & 10120 \\
\hline
\end{tabular}


According to the antenna azimuth of the base station, the base station connection graph which describes the west square in Yanji is obtained, as shown in fig. 2. Consider that the walking street in Yanji is divided into 12 cells and the number of adjacent cells in each cell is denoted by $\mathrm{m}$, as shown in table 2 , the mapping relationship between the keying coefficient and frequency point set $\mathrm{P}$ is shown in table 3 .

Based on the algorithm are given in the subsection 3.4, we calculate and solve to optimize for frequency point at the walking street west market. Consequently, computation results show as table 4, where the v3 and v9, v12 belong to the frequency point set $\mathrm{P} 2$, the rest cells is the frequency point set P1. Assumed that keying coefficient is denoted by the expression $b_{k}= \pm 1$, and then the mapping table between keying coefficient and the frequency point sets is shown in table 3 .

The results are shown in table 4 . We can see from table 4, different vertexes (that is base station) can use the same frequency as long as there is no connection between the two vertices, namely two cells is not adjacent. So the area between the two cells also doesn't have the same frequency interference.

The condition that the optimized walking street is retest is shown in figure 4 . By comparing the fig. 3 with fig. 4 , it can be obtained that before the frequency optimization for mobile network, the carrier-to-interference ratio at walking street in Yanji load is small which means that the same frequency interference is serious and network signal coverage is uneven. After the network frequency point optimized, the performance for CINR is better with less frequency interference and more even wireless network coverage at the most area of the walking street.

\section{CONCLUSIONS}

Due to the same frequency interference between the mobile communication base stations, and bad communication quality which can't meet the require ments of users, this paper proposes a mobile frequency allocation algorithm based on graph theory. The analysis for the mobile frequency points configuring scheme in Yanji verifies the efficiency of the algorithm proposed in this paper. As a consequence, not only the performance has a greater degree of improvement and has higher robustness than the other existing methods, but also it is more suitable for the application of mobile frequency point configuration.

\section{REFERENCES}

[1] Avtar Singh Buttar, Ashok Kumar Goel and Shakti Kumar. Solving 55-cell benchmark frequency assignment problem by novel nature inspired algorithm. 2014 International Conference on Signal Processing and Integrated Net works (SPIN),Noida, pp.407-411, 2014.

[2] Yu Xin. Dynamic frequency assignment based on immigrant schemes in battlefield environment.Telecommunication Engineering, 54(3),pp. 348-354,2014

[3] Wang Boyan,Lu Xiao, Chen Jie, Zhang Yanping. Design of frequent point optimization of GSM based on EGA. Computer Technology and Development,23(2),pp.23-27,2013.

[4] Cao Gen, Yang Dacheng,An Ruihong, Ye Xuan, Zheng Ruiming and Zhang Xin. An adaptive sub-band allocation scheme for dense femtocell environment. Cacun, Quintana Roo:IEEE Wireless
Communications and Networking Conference(WCNC), pp.102-107, 2011.

[5] Tan Li, Feng Zhiyong,Li Wei,Jing Zhong and Gulliver T.A. Graph coloring based spectrum allocation for femtocell downlink interference mitigation.Wireless Communications and Networking Conference(WCNC), pp.1248-1252, 2011.

[6] Sghiri,J.and Ayadi,M. An innovative approach for automatic frequency planning.2014 International Symposium on Networks,Computers and Communications,Hammamet, pp.1-5, 2014.

[7] Colin McDiarmid. On the chromatic number of random graphs. Random Structures and Algorithms, 1(4),pp.435-442, 2006.

[8] Y.Takefuji, K.C.Lee. Artificial neural net works for four-coloring map problems and K-colorability problems.IEEE Trans.Circ.Sysr,3(38), pp.326-333,1991.

[9] B.Montgomery, Dynamic coloring. Ph.D.Dissertation, West VirginiaUniversity, pp. 25-37, 2001.

[10] Jia Yongwang, Bai Lianhua, Wu Liji. The maximum planar graph 4-colorings boolean equation group.Journal of NeiMenGu Universit, Nat.Sci.Ed, 42(3), pp.37-40, 2010.

[11] Ding Chao,Fan Suohai,Lai Hongjian. The conditions of the graph coloring. Journal of jinan university, 29(1),pp.35-38,2008.

[12] Yongsheng Shi,Allen B.MacKenzie,Luiz A.DaSilva,Kaveh Ghaboosi,Matti Latva-aho. On resource reuse for cellular net works with Femto-and macrocell coexistence. Global Telecommunications Conference (GLOBECOM 2010), Miami, FL, pp.1-6, 2010. 